\title{
Robust Metallic Nanolaminates Having Phonon-Glass Thermal Conductivity
}

\author{
Francisco Alfredo García-Pastor ${ }^{1, *} \mathbb{0}$, Josué Benjamín Montelongo-Vega ${ }^{1,2}$, \\ Marco Vinicio Tovar-Padilla ${ }^{3}$, María Antonia Cardona-Castro ${ }^{1}$ and Jaime Alvarez-Quintana ${ }^{3,4, *}$ \\ 1 Centro de Investigación y de Estudios Avanzados del Instituto Politécnico Nacional Unidad Saltillo, \\ Avenida Industria Metalúrgica No. 1062. Parque Industrial, Ramos Arizpe 25900, Coahuila, Mexico; \\ jmontelo@ternium.com.mx (J.B.M.-V.); antonia.cardona@cinvestav.edu.mx (M.A.C.-C.) \\ 2 Ternium Mexico S.A. de C.V, Planta Pesquería, Carretera a Pesquería Km. 15, Ejido La Victoria, \\ Los Ramones 66650, Mexico \\ 3 Centro de Investigación en Materiales Avanzados S. C. Unidad Monterrey, Alianza Norte \# 202, \\ Autopista Mty-Aeropuerto Km.10, Apodaca 66600, Nuevo León, Mexico; opira666@gmail.com \\ 4 Genes-Group of Embedded Nanomaterials for Energy Scavenging, CIMAV-Unidad Monterrey, \\ Apodaca 66600, Nuevo León, Mexico \\ * Correspondence: francisco.garcia@cinvestav.edu.mx (F.A.G.-P.); jaime.alvarez@cimav.edu.mx (J.A.-Q.)
}

Received: 19 September 2020; Accepted: 29 October 2020; Published: 4 November 2020

\begin{abstract}
Heat transfer phenomena in multilayer structures have gained interest due to their promising use in thermal insulation and thermoelectricity applications. In such systems, nanostructuring has been used to introduce moderate interfacial density, and it has been demonstrated that interfacial thermal resistance plays a crucial role in reducing thermal conductivity $\kappa$. Nevertheless, the main constraint for actual applications is related to their tiny size because they are extremely thin to establish appreciable temperature gradients. In this work, by severe plastic deformation process of accumulative roll bonding (ARB), a $250 \mu \mathrm{m}$ thick $\mathrm{Cu}-\mathrm{Nb}$ multilayer containing more than 8000 interfaces with periods below $40 \mathrm{~nm}$ was obtained, enabling the production of bulk metallic nanolaminates with ultralow K. Multilayers present an ultralow $\mathrm{K}$ of $\sim 0.81 \mathrm{~W} / \mathrm{mK}$ at $300 \mathrm{~K}$, which is 100 times smaller than its $\mathrm{Cu}-\mathrm{Nb}$ bulk counterpart, and even lower than the amorphous lattice limit for the $\mathrm{Cu}-\mathrm{Nb}$ thin film system. By using electron diffusive mismatch model (EDMM), we argue that both electrons diffusively scattering at interface and those ballistically crossing the constituents are responsible for heat conduction in the $\mathrm{Cu}-\mathrm{Nb}$ multilayers at nanoscale. Hence, ARB $\mathrm{Cu}-\mathrm{Nb}$ multilayers are intriguing candidate materials which can prove avenues to achieve stable ultralow $\mathrm{k}$ thermal barriers for robust applications.
\end{abstract}

Keywords: nanoscale heat transfer; metallic multilayer composites; high performance thermal barriers; thermal boundary resistance; accumulative roll bonding

\section{Introduction}

Decreased efficiencies in energy conversion systems, as well as failure commonly found in power microelectronic circuits are the main motivation towards the development of new thermal dissipation and insulation materials embedded in the operating components. In this sense, multilayer structures have gained interest due to their promising use as tunable thermal conductivity materials via their interfacial thermal resistance [1,2]. Nevertheless, multilayered architecture-based devices utilizing 2D layered materials have opened a world of chances and failures. For instance, interfaces between solid layers in nanoelectronics are undesirable because they contribute significantly to the overall thermal resistance, which is a bottleneck for the further advancement of such devices. Thermal management 
in these devices has become problematic because faster and denser circuits are being packaged; as a consequence, localized areas of high heat flux are dominating the performance of electronics at both chip and board level in current nm-technologies [3-6]. On the other hand, the recent boost of research in thermoelectricity and thermal protection is closely linked to recent advances in phonon engineering via materials nanostructuring. In such applications, very low thermal conductivity materials are desirable to better engineer those systems [7-11]. To the present day, extensive experimental and theoretical investigations of thermal transport across metal-dielectric interfaces have been conducted to gain further understanding of the physics behind nanoscale thermal transport. For instance, Costescu et al. reported an ultralow $\mathrm{K}$ of $\sim 0.6 \mathrm{~W} / \mathrm{mK}$ in $\mathrm{W} / \mathrm{Al}_{2} \mathrm{O}_{3}$ nanolaminates with a high interface density [12]. Kim et al. observed a lower value of $\sim 0.3 \mathrm{~W} / \mathrm{mK}$ in $\mathrm{Ge}_{2} \mathrm{Sb}_{2} \mathrm{Te}_{5} / \mathrm{ZnS}: \mathrm{SiO}_{2}$ multilayers [13], and Chen et al. in $\mathrm{Au} / \mathrm{Si}$ multilayers found a value as low as $0.33 \mathrm{~W} / \mathrm{mK}$ via solids with highly dissimilar Debye temperatures [14]. In those systems, due to the metal region being thick compared with the energy carrier mean free paths, thermal energy transfer is mainly rendered by the electron-phonon coupling at the interface. Conversely, Goodson et al. reported a $\kappa$ of $\sim 1.2 \mathrm{~W} / \mathrm{mK}$ in Mo/Si multilayers with periods below $10 \mathrm{~nm}$; in this case, the small thickness of the metal film inhibits the electron-phonon coupling, which suppresses the electron contribution to conduction in the film-normal direction and renders phonons the dominant heat carriers [15]. Evidently, while the mechanisms related to cross-plane heat conduction in multilayers have been studied in detail previously, the main constraint for actual applications is related to their tiny size. They are too thin to establish appreciable temperature gradients and to withstand large heat fluxes. For instance, in mechanical compression refrigeration systems, thermal insulation between the interior chamber and the steel laminate exterior cabinet occurs via a thick polyurethane panel [16]. It is possible that in high-specification systems and niche applications, a thermal insulation material with adequate mechanical properties and thermal stability as part of the exterior cabinet would be more convenient. Following this idea, processing of a sample with hundreds or thousands of interfaces on a wide area with significant thickness via habitual layer-by-layer deposition techniques such as radio frequency (RF) sputtering, E-beam evaporation, atomic layer deposition (ALD), molecular-beam epitaxy (MBE), or any other would be impractical. In this work, we present a rational approach to achieve ultralow thermal conductivity bulk metallic multilayers via ultrahigh content of interfaces with potential in robust structural applications as part of the operating components. In contrast to sputtering or atomic layer deposition techniques, which only yield extremely thin specimens, we follow a processing route to obtain bulk nanolaminates by severe plastic deformation process of accumulative roll bonding (ARB) in bimetallic strips. Following this route, a $250 \mu \mathrm{m}$ thick $\mathrm{Cu}-\mathrm{Nb}$ multilayer containing more than 8000 interfaces with periods below $40 \mathrm{~nm}$ has been obtained with a value of $\sim 0.81 \mathrm{~W} / \mathrm{mK}$ at room temperature. Such value is lower than what is predicted by the amorphous limit model developed by Cahill et al. for the $\mathrm{Cu}-\mathrm{Nb}$ thin film system [17]. In fact, it is well below the value of $k$ for amorphous dielectrics such as $\mathrm{SiO}_{2}$ [17]. Moreover, by using the EDMM, it is argued that both electrons diffusively scattering at the interface and those ballistically crossing the constituents are responsible for heat conduction in the $\mathrm{Cu}-\mathrm{Nb}$ multilayers at nanoscale.

\section{Materials and Methods}

Copper and niobium are immiscible in the solid range, as seen in the phase diagram for this system [18]. This limitation arises from the differences in crystal structure between the two metals, as well as the large mismatch between the constituents' atomic radii. Therefore, such materials are good candidates for the development of bulk nanolamellar $\mathrm{Cu}-\mathrm{Nb}$ composites for robust thermal barrier applications. Starting materials consist of single-phase laminates of commercial purity $\mathrm{Cu}$ and $\mathrm{Nb}$ which are degreased, wire-brushed, and stacked. Iterating these steps increases yields to an exponential increase of the number of layers while decreasing layer thickness. A schematic representation of the general ARB process is shown in Figure 1a. ARB processing was carried out in a rolling mill with a maximum separating force of 20 metric tons, equipped with D2 steel rollers of $10 \mathrm{~cm}$ in diameter, following the procedure as described in reference [19]. Before stacking, surfaces of $\mathrm{Cu}-\mathrm{Nb}-\mathrm{Cu}$ sheets of 
500, 1000 and 500 microns thick, respectively were brushed and cleaned using acetone in an ultrasonic bath. Then, stacks were preheated in an inert atmosphere furnace at $700{ }^{\circ} \mathrm{C}$ for $4 \mathrm{~min}$, and the rolling linear speed was set at $5 \times 10^{-3} \mathrm{~m} / \mathrm{s}$. After each ARB pass, the bonded laminate was cut transversally in half using a mechanical shear. The two halves were then subjected to repetitive process of cleaning, stacking and rolling until obtaining the multilayers with ultrahigh content of interfaces.

a)
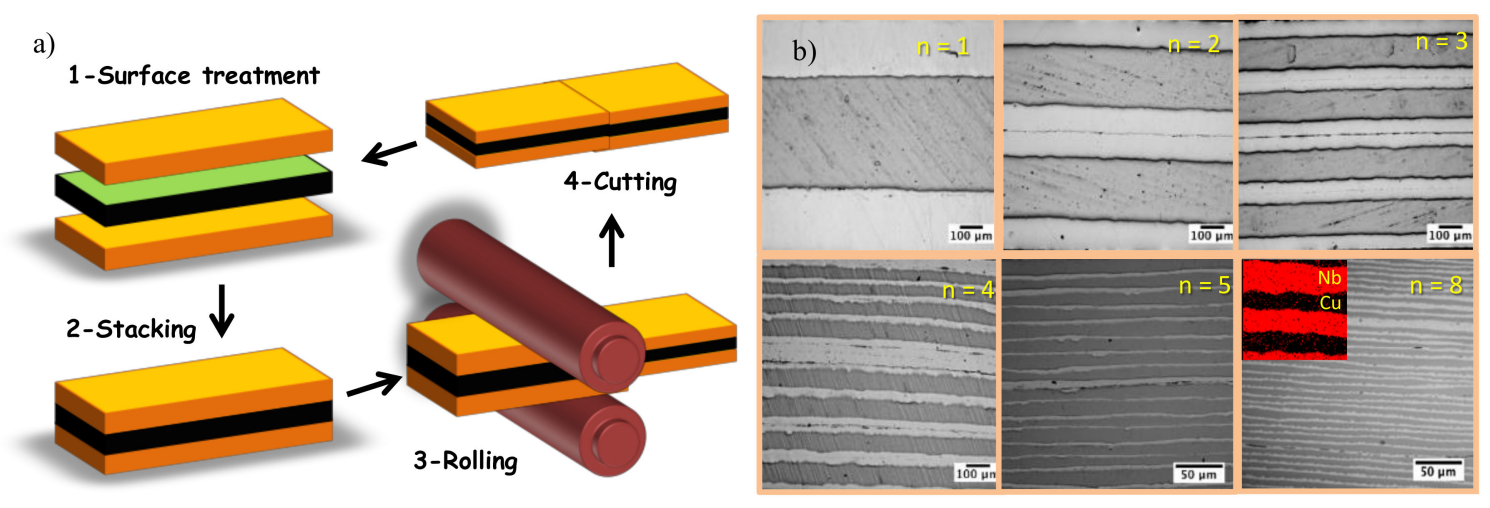

Figure 1. (a) Schematic of the ARB process, and (b) Optical images for samples with different number of ARB iterations. Inset shows the energy-dispersive-spectroscopy (EDS) chemical analysis of the $\mathrm{Nb}-\mathrm{Cu}$ multilayers.

In Figure 1b, optical images of the multilayers after eight sequential ABR processes are shown. Evidently, after each ABR stage, the number of interfaces $N$ is increased following the power law $N=2^{n}$ where $n$ stands for the process number. Based on this, the optical image with $n=8$ presents a transversal section of the sample containing 256 interfaces, which is a considerable number of interfaces. Inset shows the EDS (Oxford Instruments plc, Abingdon, Oxfordshire, UK) chemical analysis of the multilayers, $\mathrm{Cu}$ and $\mathrm{Nb}$ were identified as alternating layers. Although ARB has been applied to several different bimetal systems [20-23], the $\mathrm{Cu}-\mathrm{Nb}$ system offers negligible solid solubility between the two phases. These characteristics result in excellent microstructural stability during ARB processing, allowing production of bulk metallic nanolaminates. In this sense, intense investigations have been made into determining the effects of interface character on behavior of $\mathrm{ARB} \mathrm{Cu}-\mathrm{Nb}$ composites in terms of mechanical behavior and stability when exposed to elevated temperature [24]. However, one logical extension of the works performed here is study of $\mathrm{Cu}-\mathrm{Nb}$ nanolaminates intended for heat protection, where simultaneously high strength, thermal stability, and very low thermal conductivity will be key engineering requirements for next generation of robust thermal barrier materials. In this work, tensile strength was measured using small-scale notched tensile samples, with a cross-sectional area of $2 \mathrm{~mm}^{2}$. A Deben Microtester (Deben UK, Ltd., Woolpit, Bury St. Edmunds, UK) equipped with a $2 \mathrm{KN}$ load cell was used for tensile testing. Thermal response of the samples has been carried out via the hot-plate method. To prevent uncertainties, the measurement system was put inside of a conventional vacuum system to avoid heat loss by convection. Additionally, a radiation shield is used in order to protect the equipment from thermal fluctuations coming from the environment. Details of thermal measurements are given in the Supplementary Materials.

\section{Results}

Figure 2 shows scanning electron microscopy (SEM) images for samples with $n=11, n=12$ and $n=13$, iterations, which theoretically correspond to $N=2048, N=4096$ and $N=8192$ interfaces, respectively. It can be seen that there are well-defined interfaces formed by continuous layers of some nanometers in thickness, e.g., in sample with $n=13$, a period below $40 \mathrm{~nm}$ is identified. Insets present high-resolution transmission electron microscopy (HRTEM) images at the interfaces. Images indicate that both $\mathrm{Cu}$ and $\mathrm{Nb}$ retain their bulk stable crystal structures, resulting in a stacking sequence of $\mathrm{Cu}$ in face-centered cubic (FCC) structure and $\mathrm{Nb}$ in body-centered cubic (BCC) structure 
forming highly incoherent $\mathrm{Cu}-\mathrm{Nb}$ interfaces with few interfacial dislocations. Previous experimental investigations confirm that there is no mixing at the $\mathrm{Cu}-\mathrm{Nb}$ interface up to $873 \mathrm{~K}$; the interface texture is stable as well up to $973 \mathrm{~K}$, where a Kurdjumov-Sachs orientation relationship $(\{112\} \mathrm{Cu} \|\{112\} \mathrm{Nb})$ is the most thermally stable [24,25], which is an essential requisite to obtain highly stable interfacial thermal resistances. Besides, interface modeling based on molecular dynamics has confirmed such incoherence and low interfacial dislocation in faceted $\mathrm{FCC}(\mathrm{Cu}) / \mathrm{BCC}(\mathrm{Nb})$ interfaces [26]. Obviously, synergy between thermal and mechanical properties is indispensable for robust stable thermal barriers as part of the operating components. Metallic-based multilayered nanocomposites are recognized for their increased plastic flow strength, increased ductility, and enhanced fatigue-failure resistance in comparison to their coarse-grained constituents [27]. Compared to either pure $\mathrm{Cu}$ or $\mathrm{Nb}$ which display an ultimate tensile strength (UTS) of just 210 and $150 \mathrm{MPa}$, respectively in the annealed condition, the 13-iterations ARB-processed composite exhibits a 5-fold increase in UTS, reaching just under $1300 \mathrm{MPa}$ of tensile strength, as shown in Figure 2d. Thus, compared to the bulk constituent materials, the nanocomposite is mechanically stronger, which is particularly relevant for potential in robust structural applications. The dramatic increase in UTS is mainly related to the stability of $\mathrm{Cu}-\mathrm{Nb}$ interfaces which act as effective barriers to slip transmission [28].
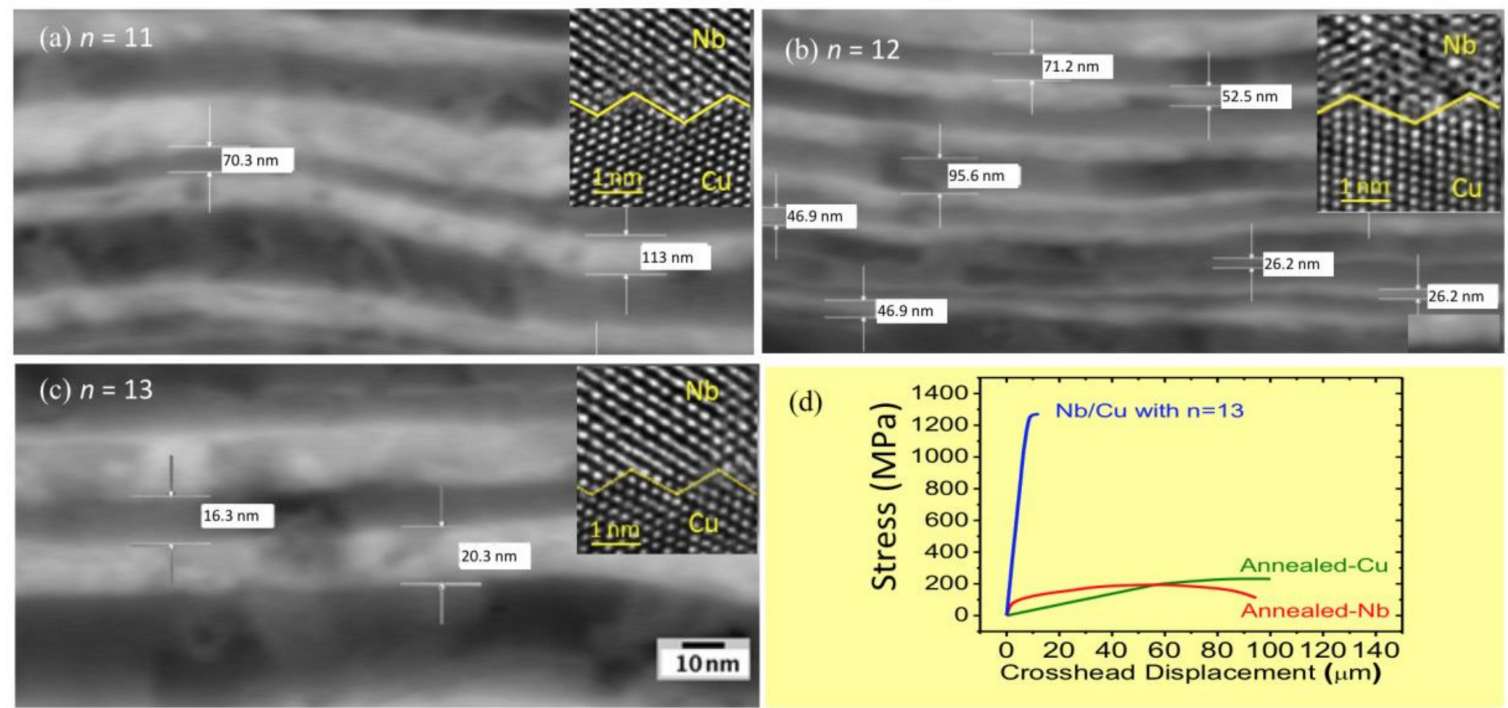

Figure 2. (a) HRTEM image of the Nb-Cu multilayers with $n=11$, (b) $n=12$ and (c) $n=13$ ARB iterations. (d) UTS for sample with $n=13$ ARB iterations as compared with their annealed constituents. Insets shows HRTEM images of the multilayers at the interface.

Previous studies suggest that interfaces between metallic materials with considerable differences in their lattice constants and structures would possess a high interfacial thermal resistance [27-31], and if combined with high interface content via multilayers, one would expect a very low $\mathrm{K}$. Figure $3 \mathrm{a}$ shows the $\Delta T-Q$ transfer curve for sample with $n=13$ iterations. Using the geometrical parameters, and the slope which represents the thermal resistance, a value of $\kappa \sim 0.81 \mathrm{~W} / \mathrm{mK}$ was obtained. By a similar procedure, the $\mathrm{k}$ of the multilayer samples from $n=1$ to $n=12$ was measured.

Figure $3 b$ shows the thermal conductivity of the ARB $\mathrm{Cu}-\mathrm{Nb}$ composites as a function of the number of interfaces. Evidently, as the number of interfaces increases, the thermal conductivity decreases drastically; this behavior makes sense because the overall interfacial resistance increases as the number of interfaces increases. According to the literature, values of $k_{C u} \approx 400 \mathrm{~W} / \mathrm{mK}$ and $k_{\mathrm{Nb}} \approx 54 \mathrm{~W} / \mathrm{mK}$ are reported for bulk $\mathrm{Cu}$ and $\mathrm{Nb}$, respectively. Therefore, by using the thermal resistor model, the effective thermal conductivity neglecting interfacial resistance effects for a two-segment slab is given by

$$
k_{\text {slab }}=\frac{2 k_{1} k_{2}}{\left(k_{1}+k_{2}\right)}
$$


where $i$ defines the metal. Based on Equation (1), an effective thermal conductivity value of around $95 \mathrm{~W} / \mathrm{mK}$ for the bulk Cu-Nb structure is expected. Nevertheless, for $n=1$ the experimentally obtained value of $\kappa$ drops drastically to $26.5 \mathrm{~W} / \mathrm{mK}$, thus significant thermal resistance effects at the interface are present. Under a physical context, contact between two surfaces occurs in a few discrete points because of the asperities and roughness present in actual surfaces. As a result, heat flux will tend to pass through the small contact points and will avoid the interstices. Depending on the surfaces' roughness in contact, temperature discontinuity at the interface between two dissimilar solids can be promoted either by the thermal contact resistance (TCR), usually with very rough surfaces whose root-mean-square roughness is greater than $50 \mu \mathrm{m}$ [31], or by the interfacial thermal resistance (ITR) with surfaces roughness ranging from some nanometers to several tens of nanometers [2,3]. Following this, the effective thermal conductivity of a two-segment slab taking into consideration both effects is given by

$$
k_{\text {ef }}^{-1}=k_{\text {slab }}^{-1}+\frac{R_{I T R}}{t_{1}}+\frac{R_{T C R}}{t_{2}}
$$

where $R_{I T R}$ and $R_{T C R}$ stand for the interfacial and contact thermal resistances, and $t_{i}$ defines the period thickness.
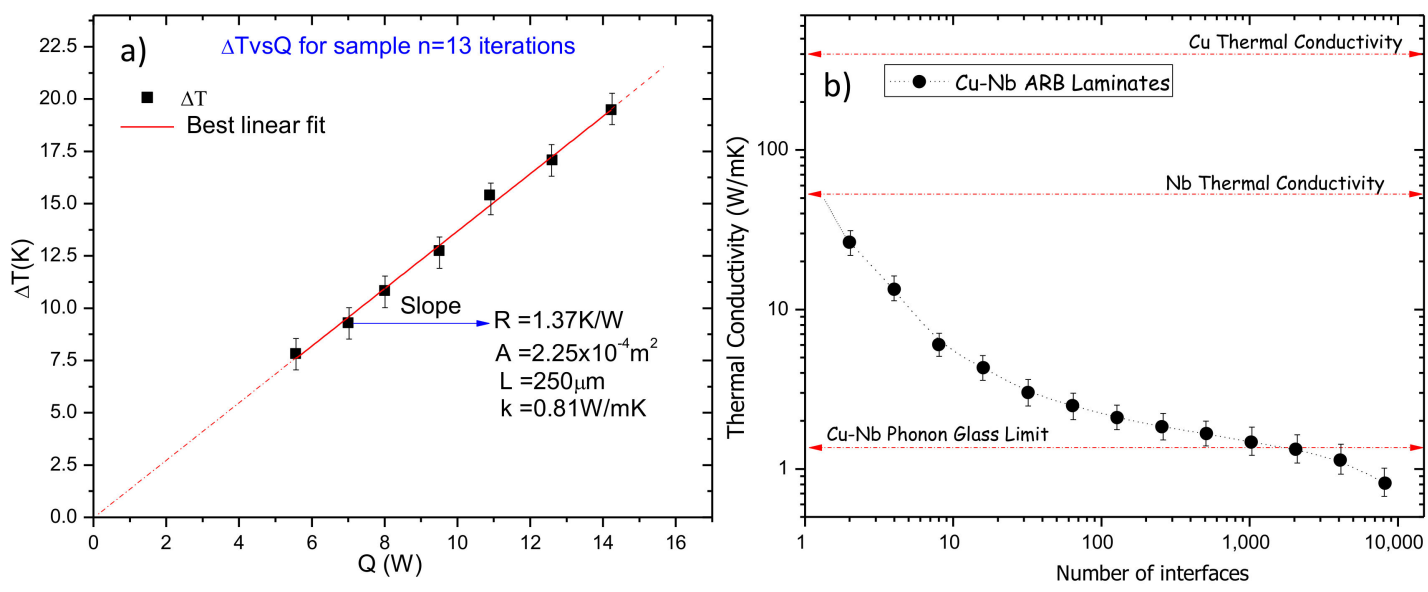

Figure 3. (a) $\Delta T-Q$ transfer curve for sample with $n=13$, and (b) $\kappa$ for samples $n=1$ to $n=13$.

Extensive experimental and theoretical studies have been carried out in the past to explain the ITR through interfaces, but the acoustic mismatch model (AMM) and the diffusive mismatch model (DMM) are the two basic models [32,33]. The AMM assumes that incident phonons at an interface undergo specular reflection or transmission and are governed by continuum mechanics, whereas in the DMM, the probability of phonon transmission to either side of the interface depends on the ratio of the density of phonon states. Pursuant to DMM, the ITR spans in a range from $10^{-7}$ to $10^{-9} \mathrm{~m}^{2} \cdot \mathrm{K} / \mathrm{W}$ [32-34]. Hence, only multilayers with period length below $10^{-9} \mathrm{~m}$ are influenced significantly in their overall thermal conductivity by the ITR in accordance with Equation (2).

Figure $4 \mathrm{a}$ shows the period length as a function of the iteration number $n$ for the ARB Cu-Nb composites. It can be seen that period length approximately spans over three scales: mm range for $1 \lesssim n \lesssim 6, \mu \mathrm{m}$ range for $7 \lesssim n \lesssim 10$ and nm range for $11 \lesssim n \lesssim 13$. Thus, only samples for $n \gtrsim 11$ must experience significant ITR effects, which means that the second term in the right side of Equation (2) tends to zero in the $\mathrm{mm}$ and $\mu \mathrm{m}$ range. Therefore, the drastic reduction observed in $\mathrm{k}$ for the ARB $\mathrm{Cu}-\mathrm{Nb}$ composites with $n \lesssim 10$ is linked to the TCR term in Equation (2). The inset in Figure 4a shows an optical image for the sample with $n=1$ iteration, contact points and voids are evident at the interfaces. Moreover, Figure $4 \mathrm{~b}-\mathrm{d}$ shows SEM images for samples with $n=2, n=4$ and $n=6$ iterations, respectively. Clearly, when pressure on the interface is increased via ARB process, the points in contact are deformed and they increase both in size and number, as highlighted by the red arrows in 
the images for samples with $n=2$ and $n=4$. Hence, the interfaces between metals become gradually in total contact because of the reduction of the surface's roughness, as shown for a sample with $n=6$.
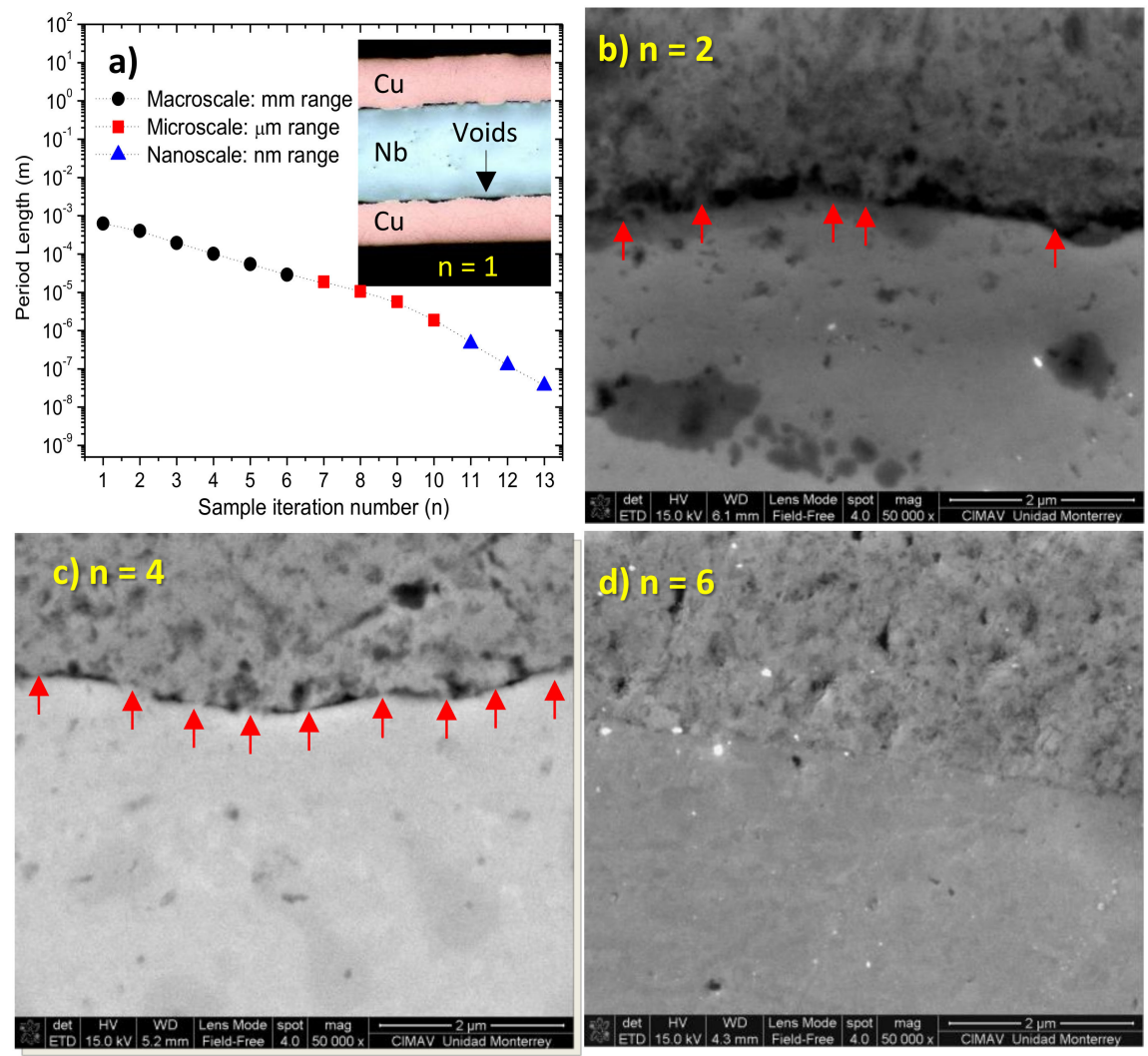

Figure 4. (a) Period length vs ARB iteration number. Inset shows sample with $n=1$ iteration, contact points and voids are evident at the interfaces. Samples with (b) $n=2$, (c) $n=4$, (d) $n=6$ ARB iterations, respectively.

\section{Discussion}

To gain a quantitative understanding of these results, we suggest the following model. Figure 5 shows an illustrative representation of actual metallic interfaces. It is considered that, for most contacts, void height is relatively smaller than its width. Because of this relative size, it is possible to neglect radial heat convection as well as heat conduction across the voids. Thus, the thermal contact resistance $R_{T C R}$ associated with this type of interface will depend on the thermal conductivity of the metals as well as the thermal conductivity of the fluid filling the voids, and it can be estimated using the method proposed by Rohsenow [35] as:

$$
\frac{1}{R_{T C R}}=\frac{\left(\frac{k_{f}}{\delta_{1}+\delta_{2}}\right)\left[\left(1-\alpha^{2}\right) C+1.1 \alpha f(\alpha)\left(\frac{1}{k_{1}}+\frac{1}{k_{2}}\right)\right]+4.26 \alpha \sqrt{n_{p}}}{\left(1-\alpha^{2}\right)\left[1-\left(\frac{k_{f}}{\delta_{1}+\delta_{2}}\right)\left(\frac{\delta_{1}}{k_{1}}+\frac{\delta_{2}}{k_{2}}\right)\right] C}
$$

The equation for $R_{T C R}$ presented in Equation (1) is a sum of two terms. Within the square brackets in the numerator (effectively, the first term) is an expression representing heat flow through the voids. Heat flow through the metallic contacts is considered in the second term. $k_{i}$ represents the thermal conductivity of the metals forming the interface, and $k_{f}$ the thermal conductivity of the fluid in the void. Likewise, $\alpha$ is the squared root of the ratio of the real area of contact $A c$ to the total contact area $A$. Besides, in Equation (3), $\delta_{i}$ is a parameter that expresses the equivalent idealized gap thickness in terms of the average heights $\overline{Z_{i x j}}$ and $\overline{Z_{i y j}}$ of the voids of actual surfaces in the $x$ and $y$ direction respectively, as shown in Figure 5. The subscript $i$ refers to metal 1 or 2 , and $j$ refers to the number of 
the void. The number of contact points per unit area $n_{p}$ is obtained by dividing the product of the number of contacts on each pair of profiles in the $x$ and $y$ directions, i.e., $n_{x i} n_{y i}$ over an area defined by $l_{x} l_{y}$. The term $C$ is a factor which is only used to compact Equation (3), and it is given by

$$
C=\frac{1+4.26 \sqrt{n_{p}}\left(\frac{\delta_{1}}{\alpha}\right)}{k_{1}}+\frac{1+4.26 \sqrt{n_{p}}\left(\frac{\delta_{2}}{\alpha}\right)}{k_{2}}
$$

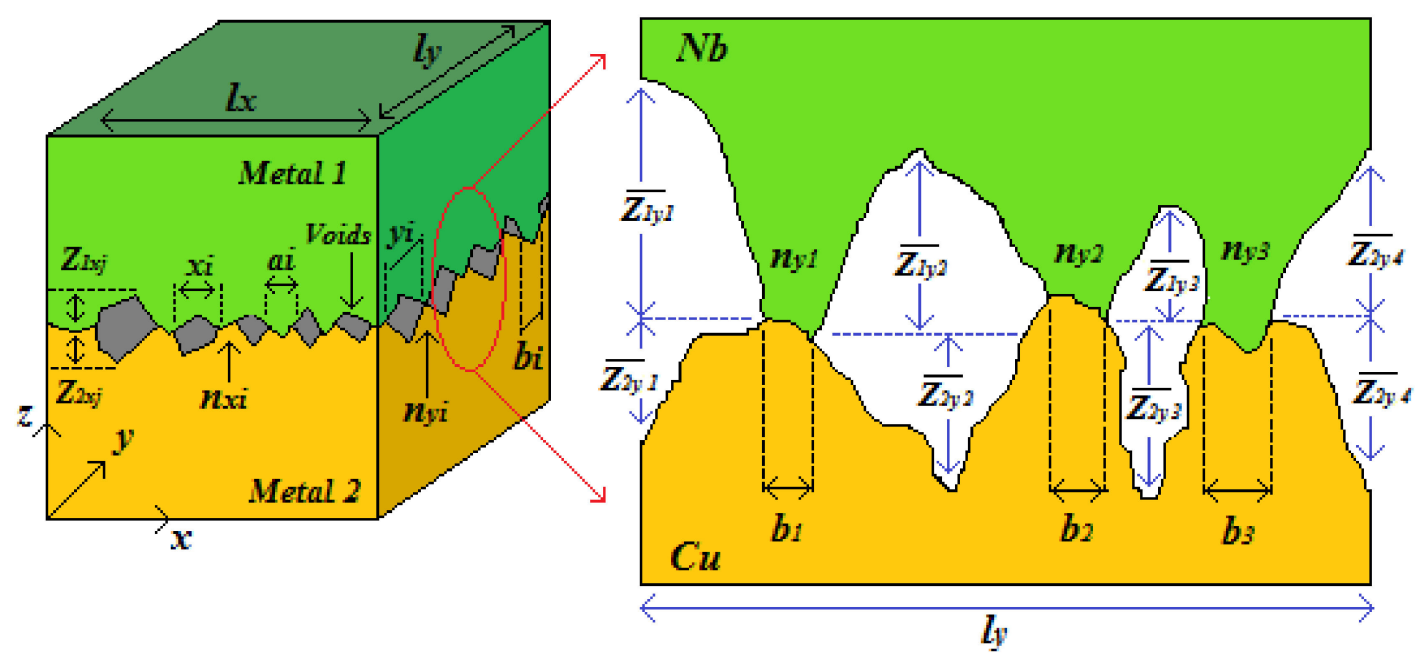

Figure 5. Illustrative model of an interface between two contacting metallic surfaces for samples with period lengths in the $\mathrm{mm}$ range, i.e., $1 \lesssim n \lesssim 6$. In the figure, $n_{x i}, a_{i}, x_{i}$ and $n_{y i}, b_{i}, y_{i}$, represent the number of contact points, size of the contact points and size of the voids in the $x$ and $y$ directions, respectively. $\overline{Z_{i x j}}$ and $\overline{Z_{i y j}}$ are the void average height in the $x$ and $y$ directions, respectively.

Finally, with these quantities $\alpha, n_{p}$ and $\delta_{i}$, the magnitude of $R_{T C R}$ can be predicted from Equation (3). Experimental thermal resistance at interface for the whole set of samples is shown in Figure 6a as open symbols. Such values were extracted from the effective thermal conductivity data shown in Figure 3 along with Equation (2). The black solid line and open circle symbols represent the modeling and experimental data respectively for samples with $1 \lesssim n \lesssim 6$ iterations. Evidently, beyond $n \gtrsim 7$, the model given by Equation (3) deviates significantly. It is worth mentioning that because voids at the interface diminish as the iteration number increases, it was possible to accomplish reasonably good modeling results only for samples with $n \lesssim 6$.

The problem with Equation (3) is related to the function $f(\alpha)$; for practical purposes, the ratio of the squared root of the effective area of contact to the total contact area must be $\alpha \lesssim 0.1$. Under this condition, $f(\alpha) \approx 1$, otherwise, $f(\alpha)$ drops drastically well below 1 . The physical meaning of $f(\alpha) \ll 1$ implies that the effective area of contact to the total contact are very similar, thus $\alpha \approx 1$, i.e., no more voids or a very high contact points density; hence, the model fails in such a situation. To overcome such condition, the first term in Equation (3) can be neglected since flow across the voids is neglected as well. By using such math artifice, Equation (3) becomes independent of $f(\alpha)$, and the model can be applied for samples with periods at the microscale where the interfaces have much better contact, i.e., $7 \lesssim n \lesssim 10$. In Figure $6 \mathrm{a}$, square open symbols and a red solid line represent the modeling and experimental data, respectively, for samples with $7 \lesssim n \lesssim 10$ iterations. Evidently, beyond $n \gtrsim 11$, the model again deviates significantly, and it is unable to predict accurately the contact resistance for samples with periods at the nanoscale, i.e., $11 \lesssim n \lesssim 13$. The detailed procedures of calculations of the $R_{T C R}$ at the macro/microscale regime are given in the Supplementary Materials (Pages 5-11, Equations (1)-(10)). 

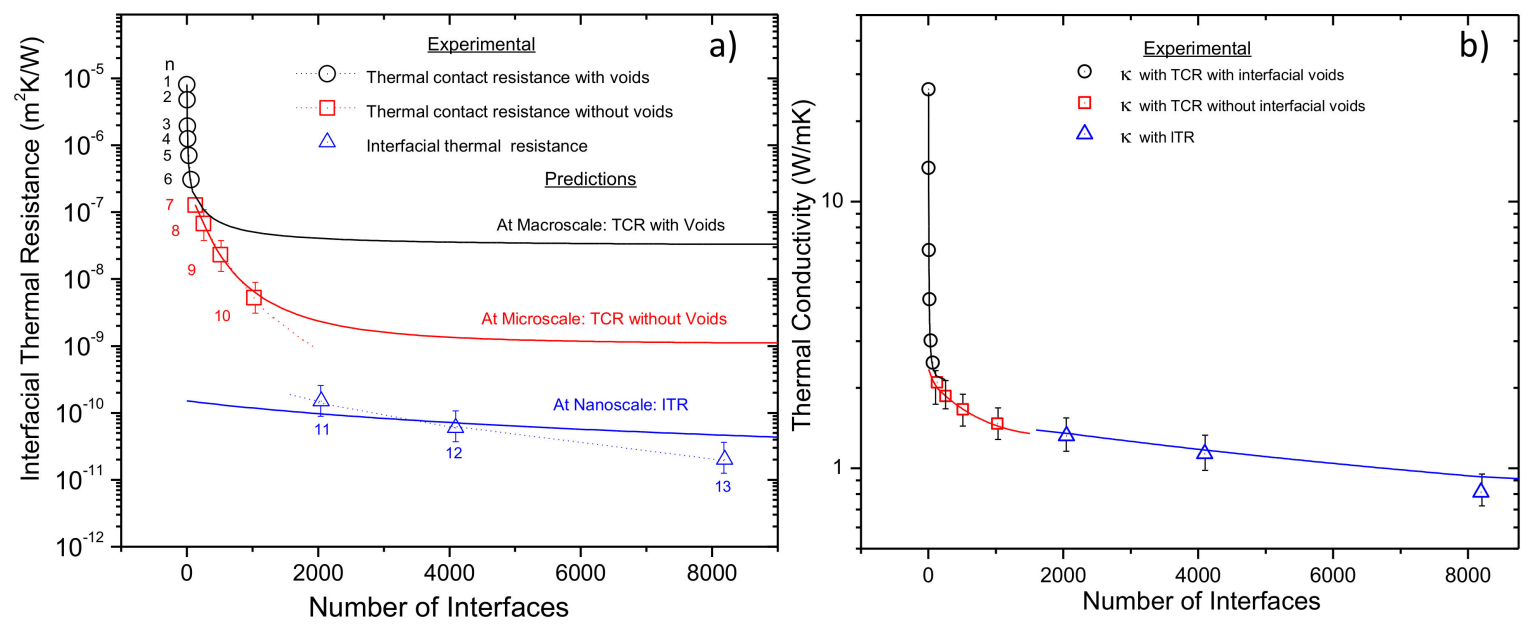

Figure 6. (a) Interface-dependent thermal resistance at interface, and (b) Interface-dependent thermal conductivity for ARB samples. In both plots, solid lines are the calculated $R_{\text {eITR }}, R_{T C R}$ and $\mathrm{k}$ values which are reasonably consistent with the experimental data.

At the nanoscale regime, when the films' thicknesses are relatively larger than the electron mean free path, pure diffusion is adequate to model thermal transport inside them. Therefore, assuming perfect contact between the two metals, it is also possible to assume that electrons are in equilibrium on each side of the interface, and just at the interface, electrons will be scattered following a probability that depends solely on the properties of the metals. Nevertheless, when the films' thicknesses are comparable with or smaller than the electron mean free path, thermal transport must be modeled as purely ballistic. If we take into consideration that the average period length for $\mathrm{Cu}-\mathrm{Nb}$ composites with $n \gtrsim 11$ ranges from $400 \mathrm{~nm}$ to $40 \mathrm{~nm}$, while $\mathrm{Cu}$ and $\mathrm{Nb}$ are both free-electron metals with mean free paths of $\sim 86$ and $\sim 8 \mathrm{~nm}$, respectively, then the electron diffusive mismatch model EDMM assumptions are adequately describing electron transport across the aforementioned interfaces. In the EDMM approach [30], the interfacial thermal resistance is given by

$$
\frac{1}{R_{I T R}}=\frac{1}{4}\left(\frac{Z_{1} Z_{2}}{Z_{1}+Z_{2}}\right)
$$

where $Z_{i}=C_{e i} v_{F i}$; here, $C_{e i}$ is the electronic heat capacity and $v_{F i}$ is the electron Fermi velocity of the metal on side $i$. Evidently, electrons move ballistically from the copper side to the niobium side, where then they scatter diffusively depending on the surface roughness. Hence, the effective thermal interface resistance is thus the sum of the contribution of both the electrons ballistically traveling across the $\mathrm{Cu}-\mathrm{Nb}$ system and those diffusively scattering at the interface. Therefore, the effective ITR is given by

$$
\frac{1}{R_{\text {eITR }}}=\frac{1}{R_{\text {ITR-B }}}(\beta)+\frac{1}{R_{I T R-D}}(1-\beta)
$$

where $\beta=e^{\left(-d / \lambda_{e}\right)}$ is a term derived from ballistic diffusive equations and describes the exponential decay of heat flux across interface [36]. $R_{(I T R-B)}$ and $R_{(I T R-D)}$ stand for the ballistic and diffusive ITR contributions, respectively. Values of the parameters and calculations of the $R_{\text {eITR }}$ are given in the Supplementary Materials (Pages 11-12, Equations (11)-(19)). In Figure 6a, blue open triangle symbols show the experimental data of $\mathrm{Cu}-\mathrm{Nb}$ multilayers with nanometric period lengths. Such data are in reasonable agreement according to the EDMM results given by the blue solid line. Clearly, from Figure 6a, the thermal resistance at the interface depends on the scale regime. At macro/microscale regimes $R_{T C R}$ lies between $10^{-6}$ and $10^{-8} \mathrm{~m}^{2} \cdot \mathrm{K} / \mathrm{W}$; whereas at nanoscale regime, $R_{T C R}$ lies between $10^{-9}$ and $10^{-11} \mathrm{~m}^{2} \cdot \mathrm{K} / \mathrm{W}$. By examining the literature, at macro/microscale, $R_{T C R}$ in metal-metal interfaces depends strongly on several factors such as surface roughness, asperity and pressure. 
In general, as the contact pressure increases, $R_{T C R}$ decreases, and as the roughness of the surface decreases, $R_{T C R}$ decreases. Fletcher et al. reported $R_{T C R}$ values between layers of stacked aluminum alloys ranging from $10^{-4}$ to $10^{-6} \mathrm{~m}^{2} \mathrm{~K} / \mathrm{W}$ under moderate contact pressure, but lower values can be accomplished for smooth surfaces under high pressures [37]. At the nanoscale, multilayer metallic composites in which electrons in constituent metals have considerable different mean free paths may provide a quantitative comparison with our $R_{\text {eITR }}$ experimental values. Wilson and Cahill reported a $R_{\text {ITR }} \approx 8.2 \times 10^{-11} \mathrm{~m}^{2} \cdot \mathrm{K} / \mathrm{W}$ across Pd-Ir interfaces at room temperature [29], whereas Gundrum et al. reported a $R_{I T R} \approx 2.7 \times 10^{-10} \mathrm{~m}^{2} \cdot \mathrm{K} / \mathrm{W}$ across $\mathrm{Cu}$-Al interfaces [30]. In the present work, for $\mathrm{Cu}-\mathrm{Nb}$ composites with $n=11, n=12$ and $n=12$, the experimental interfacial thermal resistances are $1.5 \times 10^{-10} \mathrm{~m}^{2} \cdot \mathrm{K} / \mathrm{W}, 6.2 \times 10^{-11} \mathrm{~m}^{2} \cdot \mathrm{K} / \mathrm{W}$ and $2.2 \times 10^{-11} \mathrm{~m}^{2} \cdot \mathrm{K} / \mathrm{W}$, respectively. Thus, our experimental results are in reasonable agreement with previously reported metallic interfaces, but with significantly larger (250 micrometers versus 1-2 micrometers in [30]) and more mechanically robust samples. Besides, for the sample with $n=13$ which corresponds to a period length below $40 \mathrm{~nm}$, the EDMM approach predicts a value of $5.4 \times 10^{-11} \mathrm{~m}^{2} \cdot \mathrm{K} / \mathrm{W}$, hence experimental and predicted values are similar. By using the predicted $R_{T C R}$ and $R_{\text {eITR }}$ data along with Equation (2), the effective thermal conductivity of the $\mathrm{Cu}-\mathrm{Nb}$ composites has been estimated. Figure $6 \mathrm{~b}$ shows the modeling results, and experimental data as solid lines and open symbols, respectively. It is worth highlighting the ultra-low thermal conductivity value of $k_{(\mathrm{C} u-\mathrm{Nb})} \approx 0.81 \mathrm{~W} / \mathrm{mK}$ obtained for the sample with $n=13$ iterations. At this level, the term "ultra-low" is used to describe a $\kappa$ value lower than that predicted by the minimum $\kappa$ model presented by Cahill et al. [17] Therefore, the existence of a lower limit to the thermal conductivity of disordered crystals based on the idea that lattice vibrations in those solids are essentially the same as those of an amorphous solid can be approximated by

$$
k_{\min }=0.403 k_{B} n_{a}^{2 / 3}\left(2 v_{T}+v_{L}\right)
$$

where $v_{T}$ and $v_{L}$ are the transversal and longitudinal speeds of the sound, respectively, $n_{a}$ is the number of density of atoms and $k_{B}$ the constant of Boltzmann. For comparison, Table 1 shows the minimum thermal conductivity values predicted by Equation (7), as well as the reported experimental thermal conductivity for amorphous bulk materials [17]. Clearly, the thermal conductivity of $0.81 \mathrm{~W} / \mathrm{mK}$ at room temperature for the sample with $n=13$ is even lower than the $0.95 \mathrm{~W} / \mathrm{mK}$ predicted for the amorphous lattice limit of the $\mathrm{Cu}-\mathrm{Nb}$ thin film system. In fact, it is determined to be well below that of the amorphous dielectrics like $\mathrm{Al}_{2} \mathrm{O}_{3}$ or $\mathrm{SiO}_{2}$, and about 100 times lower than the $\mathrm{Cu}-\mathrm{Nb}$ system with $n=0$ iterations.

Table 1. Predicted values for the lower limit to thermal conductivity of amorphous materials.

\begin{tabular}{|c|c|c|c|c|c|}
\hline Sample & $\begin{array}{c}\left.{ }^{n}{ }^{28} \mathrm{~m}^{-3}\right) \\
\text { (1) }\end{array}$ & $\begin{array}{c}v_{T} \\
(\mathrm{~m} / \mathrm{s})\end{array}$ & $\begin{array}{c}v_{\mathrm{L}} \\
(\mathrm{m} / \mathrm{s})\end{array}$ & $\begin{array}{c}K_{\min } \\
(\mathrm{W} / \mathrm{mK})\end{array}$ & $\begin{array}{c}K_{\exp } \\
(W / m K)\end{array}$ \\
\hline $\mathrm{SiO}_{2}$ & 6.63 & 3740 & 5980 & 1.21 & 1.35 \\
\hline $\mathrm{Al}_{2} \mathrm{O}_{3}$ & 10.89 & 5800 & 9900 & 2.71 & 2.76 \\
\hline $\mathrm{Cu}$ & 8.47 & 3720 & 4720 & 1.3 & - \\
\hline $\mathrm{Nb}$ & 5.56 & 2092 & 5068 & 0.76 & - \\
\hline $\mathrm{a}-\mathrm{Cu} / \mathrm{Nb}$ System & - & - & - & 0.95 & \\
\hline $\mathrm{Cu}-\mathrm{Nb}(\mathrm{n}=0)$ & - & - & - & - & 92.5 \\
\hline $\mathrm{Cu}-\mathrm{Nb}(\mathrm{n}=13)$ & - & - & - & - & 0.81 \\
\hline
\end{tabular}

\section{Conclusions}

In conclusion, we obtained by severe plastic deformation process of accumulative roll bonding (ARB) a $250 \mu \mathrm{m}$ thick $\mathrm{Cu}-\mathrm{Nb}$ multilayer containing more than 8000 interfaces with periods below $40 \mathrm{~nm}$. Such a result enables the production of bulk metallic nanolaminates with ultralow thermal conductivity values of around $0.81 \mathrm{~W} / \mathrm{mK}$ at room temperature which is much lower than that of the amorphous dielectrics like $\mathrm{SiO}_{2}$. The large differences in electron mean free paths in $\mathrm{Cu}$ and $\mathrm{Nb}$ 
along with the thermodynamic immiscibility of these materials make them an ideal system to study the role of electron thermal transport properties on thermal conductance across metallic interfaces. We report an experimental value of $2.2 \times 10^{-11} \mathrm{~m}^{2} \cdot \mathrm{K} / \mathrm{W}$ for ITR between $\mathrm{Cu}$ and $\mathrm{Nb}$ interfaces at room temperature for the sample with $40 \mathrm{~nm}$ period length. Although, $R_{I T R}$ values obtained by the EDMM model do not match accurately the estimated $R_{I T R}$ values they give an approach about the trend of the $R_{\text {ITR }}$ as $\mathrm{n}$ increases. It is well known that such model is limited to single crystal metallic thin films, and this could be the main reason for the lack in the data fit.

The results presented are adequately described using the electronic diffuse mismatch model EDMM. This indicates that the EDMM mechanisms are acceptable for describing electron transport through the $\mathrm{Cu}-\mathrm{Nb}$ interfaces. Besides, our results are comparable to previously reported values on Al-Cu and Pd-Ir interfaces. In contrast to similar low-thermal conductivity laminates such as the ones reported in References [12,14,30], the ARB processing route yields bulk nanolaminates with remarkable mechanical strength. Not only that, but the thickness is two orders of magnitude larger in the processed samples reported in this paper, compared to samples obtained by sputtering or atomic layer deposition. Therefore, the combination of microstructural and thermal stability, high strength, and ultralow thermal conductivity make ARB metallic nanolaminates intriguing candidate materials for structural applications where the current thermal barriers based on multilayers only available in thin-film form would prove inadequate.

Supplementary Materials: The following are available online at http://www.mdpi.com/1996-1944/13/21/4954/s1, Figure S1: (a) Schematic of the ARB process, and (b) Optical images for samples with a different number of ARB iterations. Inset shows the EDS chemical analysis of the $\mathrm{Nb}-\mathrm{Cu}$ multilayers. Figure S2: (a) TEM image of the $\mathrm{Nb} C u$ multilayers with $n=11,(\mathbf{b}) n=12$ and (c) $(n=13$ ARB iterations. (d) UTS for sample with $n=13$ ARB iterations as compared with their annealed constituents. Insets shows HRTEM images of the multilayers at the interface. Figure S3: (a) Illustrative and (b) actual experimental set up for thermal conductivity measurements. Figure S4: $\Delta \mathrm{T}-\mathrm{Q}$ transfer curve for reference sample. Figure S5: $\Delta \mathrm{T}-\mathrm{Q}$ transfer curve for sample with $n=13$ iterations. Figure S6: Illustrative model of an interface between two contacting metallic surfaces for samples with period lengths in the $\mathrm{mm}$ range, i.e., $1 \lesssim n \lesssim 6$. In the figure, $n_{x i}, a_{i}, x_{i}$ and $n_{y i}, b_{i}, y_{i}$, represent the number of contact points, size of the contact points and size of the voids in the $x$ and $y$ direction, respectively. Figure S7: (a) Period length vs. iteration number $n$ for the ARB Cu-Nb composites, and (b) $n=2$, (c) $n=4$, (d) $n=6$ present the interface evolution. Figure S8: Size of the contact points in the $x$ and $y$ directions, respectively. Figure S9: Average heights $\overline{Z_{i x j}}$, and $\overline{Z_{i y j}}$ of the voids of actual surfaces in the $x$ and $y$ directions, respectively. Figure S10: Size of the voids in the $x$ and $y$ directions, respectively. Figure S11: Number of contact points per unit area $n$. Figure S12. Size-dependent thermal conductivity for $\mathrm{Cu}$ and $\mathrm{Nb}$ polycrystalline films. Solid lines represent the results obtained from model reported in reference [12], whereas solid points represent the existing experimental data for $\mathrm{Cu}$. Table S1: Parameters used for sample with $\mathrm{n}=2$ iterations. Table S2: Calculated parameters for $\mathrm{Cu}$ and $\mathrm{Nb}$ using the free electron model. Table S3: Calculated data for the interface thermal resistance between copper and niobium interfaces. Table S4: Effective thermal interface resistance calculations for samples with $\mathrm{n}=11, \mathrm{n}=12$ and $\mathrm{n}=13$ iterations. Table S5. Predicted values for the lower limit to thermal conductivity of amorphous materials. Table S6. Summary of typical uncertainty of measuring parameters. Determination of the interface physical properties for actual surfaces such as real area in contact, the number of contact points per unit area, and the average thickness of the void gaps via image analysis, as well as calculation of the thermal contact resistance $R_{T C R}$, pages 5-11, Equations (1)-(10). Determinations of the materials properties $(\mathrm{Cu}$ and $\mathrm{Nb})$ such as electron mean free path, density of states, Fermi velocity, and transmission coefficient, as well as calculation of the interfacial thermal resistance $R_{I T R}$, pages 11-12, Equations (11)-(19). Detailed discussion of the EDMM and TCR approaches, as well as calculations of the minimum thermal conductivity, pages 13-14. Measurement uncertainty assessment, pages 15-16. References [38-42] are cited in the supplementary materials.

Author Contributions: Conceptualization, methodology, resources, supervision, writing-original draft, writing-review \& editing, J.A.-Q. and F.A.G.-P.; investigation, visualization, data curation, M.V.T.-P. and J.B.M.-V.; investigation, methodology, visualization, data curation, writing-original draft, M.A.C.-C. All authors have read and agreed to the published version of the manuscript.

Funding: This work was supported by the Mexican Council for Science and Technology-Conacyt Mexico, through the Grant for fundamental research (national issues) No.1358

Acknowledgments: M.V.T.-P. and J.B.M.-V. thank Conacyt Mexico for postgraduate scholarships. M.A.C.-C. thanks Conacyt Mexico for postdoctoral fellowship 2020-2021.

Conflicts of Interest: The authors declare no conflict of interest. 


\section{Nomenclature}

\begin{tabular}{|c|c|}
\hline ARB & accumulative roll bonding \\
\hline SPD & severe plastic deformation \\
\hline EDMM & electron diffusive mismatch model \\
\hline$k$ & thermal conductivity \\
\hline $\mathrm{N}$ & number of interfaces \\
\hline$n$ & number of ARB passes \\
\hline HRTEM & high-resolution transmission electron microscopy \\
\hline Q & heat \\
\hline$k_{C u}$ & copper thermal conductivity \\
\hline$k_{N b}$ & niobium thermal conductivity \\
\hline$k_{\text {slab }}$ & two-segment bimetallic thermal conductivity \\
\hline$R_{\text {ITR }}$ & interfacial thermal resistance \\
\hline$R_{T C R}$ & thermal contact resistance \\
\hline AMM & acoustic mismatch model \\
\hline DMM & diffusive mismatch model \\
\hline$k_{i}$ & thermal conductivity of the metals forming the interface \\
\hline$i$ & subscript to refer to either metal 1 or 2 \\
\hline$k_{f}$ & thermal conductivity of the fluid in the void \\
\hline$\alpha$ & squared root of the ratio of the real area of contact to the contact area \\
\hline Ac & real area of contact \\
\hline A & total contact area \\
\hline$\delta_{i}$ & equivalent idealized gap thickness in terms of average height \\
\hline$\overline{Z_{i x j}}, \overline{Z_{i y j}}$ & average height of voids \\
\hline$j$ & subscript index for void number \\
\hline$n_{p}$ & number of contact points per unit area \\
\hline$C_{e i}$ & electronic heat capacity of metal $i$ \\
\hline $\mathrm{v}_{\mathrm{Fi}}$ & electron Fermi velocity of metal $i$ \\
\hline$\beta$ & term describing the exponential decay of heat flux across the interface \\
\hline$R_{\text {ITR-B }}$ & ballistic ITR contribution \\
\hline$R_{\text {ITR-D }}$ & diffusive ITR contribution \\
\hline$k_{\min }$ & thermal conductivity lower limit \\
\hline$v_{T}$ & transversal speed of sound \\
\hline$v L$ & longitudinal speed of sound \\
\hline$n_{a}$ & number of density of atoms \\
\hline$k_{B}$ & constant of Boltzmann \\
\hline$R_{\text {eITR }}$ & effective interfacial thermal resistance \\
\hline$\lambda_{e}$ & effective mean free path of electrons \\
\hline $\mathrm{d}$ & half metallic lamellae thickness \\
\hline
\end{tabular}

\section{References}

1. Chen, G. Thermal conductivity and ballistic-phonon transport in the cross-plane direction of superlattices. Phys. Rev. B 1998, 57, 14958-14973. [CrossRef]

2. Cahill, D.G.; Ford, W.K.; Goodson, K.E.; Mahan, G.D.; Majumdar, A.; Maris, H.J.; Merlin, R.; Phillpot, S.R. Nanoscale thermal transport. J. Appl. Phys. 2003, 93, 793-818. [CrossRef]

3. Koh, Y.K.; Bae, M.-H.; Cahill, D.G.; Pop, E. Heat Conduction across Monolayer and Few-Layer Graphenes. Nano Lett. 2010, 10, 4363-4368. [CrossRef] [PubMed]

4. Choi, D.; Poudel, N.; Cronin, S.B.; Shi, L. Effects of basal-plane thermal conductivity and interface thermal conductance on the hot spot temperature in graphene electronic devices. Appl. Phys. Lett. 2017, 110, 073104. [CrossRef]

5. Moore, A.L.; Shi, L. Emerging challenges and materials for thermal management of electronics. Mater. Today 2014, 17, 163-174. [CrossRef] 
6. Yang, J.; Ziade, E.; Maragliano, C.; Crowder, R.; Wang, X.; Stefancich, M.; Chiesa, M.; Swan, A.K.; Schmidt, A.J. Thermal conductance imaging of graphene contacts. J. Appl. Phys. 2014, 116, 023515. [CrossRef]

7. Zhou, Y.; Zhao, L.-D. Promising Thermoelectric Bulk Materials with 2D Structures. Adv. Mater. 2017, 29, 1702676. [CrossRef]

8. Zhao, L.-D.; Lo, S.-H.; Zhang, Y.; Sun, H.; Tan, G.; Uher, C.; Wolverton, C.M.; Dravid, V.P.; Kanatzidis, M.G. Ultralow thermal conductivity and high thermoelectric figure of merit in SnSe crystals. Nat. Cell Biol. 2014, 508, 373-377. [CrossRef]

9. Cao, Y.Q.; Zhao, X.B.; Zhu, T.J.; Zhang, X.B.; Tu, J.P. Syntheses and thermoelectric properties of $\mathrm{Bi}_{2} \mathrm{Te}_{3} / \mathrm{Sb}_{2} \mathrm{Te}_{3}$ bulk nanocomposites with laminated nanostructure. Appl. Phys. Lett. 2008, 92, 143106. [CrossRef]

10. Kim, S.I.; Lee, K.H.; Mun, H.A.; Kim, H.S.; Hwang, S.W.; Roh, J.W.; Yang, D.J.; Shin, W.H.; Li, X.S.; Lee, Y.H.; et al. Dense dislocation arrays embedded in grain boundaries for high-performance bulk thermoelectrics. Science 2015, 348, 109-114. [CrossRef]

11. Ma, Y.; Hao, Q.; Poudel, B.; Lan, Y.; Yu, B.; Wang, D.; Chen, G.; Ren, Z. Enhanced Thermoelectric Figure-of-Merit in p-Type Nanostructured Bismuth Antimony Tellurium Alloys Made from Elemental Chunks. Nano Lett. 2008, 8, 2580-2584. [CrossRef]

12. Costescu, R.M.; Cahill, D.G.; Fabreguette, F.H.; Sechrist, Z.A.; George, S.M. Ultra-Low Thermal Conductivity in W/Al2O3 Nanolaminates. Science 2004, 303, 989-990. [CrossRef]

13. Kim, E.-K.; Kwun, S.-I.; Lee, S.-M.; Seo, H.; Yoon, J.-G. Thermal boundary resistance at $\mathrm{Ge}_{2} \mathrm{Sb}_{2} \mathrm{Te}_{5} /$ $\mathrm{ZnS}: \mathrm{SiO}_{2} \mathrm{Ge}_{2} \mathrm{Sb}_{2} \mathrm{Te}_{5} / \mathrm{ZnS}: \mathrm{SiO}_{2}$ interface. Appl. Phys. Lett. 2000, 76, 3864-3866. [CrossRef]

14. Dechaumphai, E.; Lu, D.; Kan, J.J.; Moon, J.; Fullerton, E.E.; Liu, Z.; Chen, R. Ultralow Thermal Conductivity of Multilayers with Highly Dissimilar Debye Temperatures. Nano Lett. 2014, 14, 2448-2455. [CrossRef] [PubMed]

15. Li, Z.; Tan, S.; Bozorg-Grayeli, E.; Kodama, T.; Asheghi, M.; Delgado, G.; Panzer, M.; Pokrovsky, A.; Wack, D.; Goodson, K.E. Phonon Dominated Heat Conduction Normal to Mo/Si Multilayers with Period below $10 \mathrm{~nm}$. Nano Lett. 2012, 12, 3121-3126. [CrossRef]

16. Dalkilic, A.S.; Celen, A.; Çebi, A.; Wongwises, S. Effect of Refrigerant Type and Insulation Thickness on Refrigeration Systems of Land and Sea Vehicles. Strojniški Vestnik J. Mech. Eng. 2016, 62, 252. [CrossRef]

17. Cahill, D.G.; Watson, S.K.; Pohl, R.O. Lower limit to the thermal conductivity of disordered crystals. Phys. Rev. B 1992, 46, 6131-6140. [CrossRef]

18. Okamoto, H. Cu-Nb (Copper-Niobium). J. Phase Equilibria Diffus. 2012, 33, 344. [CrossRef]

19. Saito, Y.; Utsunomiya, H.; Tsuji, N.; Sakai, T. Novel ultra-high straining process for bulk materials-development of the accumulative roll-bonding (ARB) process. Acta Mater. 1999, 47, 579-583. [CrossRef]

20. Yasuna, K.; Terauchi, M.; Otsuki, A.; Ishihara, K.; Shingu, P. Formation of nanoscale Fe/Ag multilayer by repeated press-rolling and its layer thickness dependence of magnetoresistance. Mater. Sci. Eng. A 2000, 285, 412-417. [CrossRef]

21. Knezevic, M.; Nizolek, T.; Ardeljan, M.; Beyerlein, I.J.; Mara, N.A.; Pollock, T.M. Texture evolution in two-phase $\mathrm{Zr} / \mathrm{Nb}$ lamellar composites during accumulative roll bonding. Int. J. Plast. 2014, 57, 16-28. [CrossRef]

22. Chang, H.; Zheng, M.; Gan, W.; Wu, K.; Maawad, E.; Brokmeier, H. Texture evolution of the Mg/Al laminated composite fabricated by the accumulative roll bonding. Scr. Mater. 2009, 61, 717-720. [CrossRef]

23. Hosseini, M.; Pardis, N.; Manesh, H.D.; Abbasi, M.; Kim, D.-I. Structural characteristics of Cu/Ti bimetal composite produced by accumulative roll-bonding (ARB). Mater. Des. 2017, 113, 128-136. [CrossRef]

24. Mara, N.A.; Beyerlein, I.J. Review: Effect of bimetal interface structure on the mechanical behavior of $\mathrm{Cu}-\mathrm{Nb}$ fcc-bcc nanolayered composites. J. Mater. Sci. 2014, 49, 6497-6516. [CrossRef]

25. Carpenter, J.; Zheng, S.; Zhang, R.; Vogel, S.; Beyerlein, I.; Mara, N. Thermal stability of Cu-Nb nanolamellar composites fabricated via accumulative roll bonding. Philos. Mag. 2013, 93, 718-735. [CrossRef]

26. Kang, K.; Wang, J.; Zheng, S.J.; Beyerlein, I.J. Minimum energy structures of faceted, incoherent interfaces. J. Appl. Phys. 2012, 112, 73501. [CrossRef]

27. Clemens, B.M.; Eesley, G.L.; Paddock, C.A. Time-resolved thermal transport in compositionally modulated metal films. Phys. Rev. B 1988, 37, 1085-1096. [CrossRef]

28. Wang, J.; Hoagland, R.; Misra, A. Mechanics of nanoscale metallic multilayers: From atomic-scale to micro-scale. Scr. Mater. 2009, 60, 1067-1072. [CrossRef] 
29. Wilson, R.B.; Cahill, D.G. Experimental Validation of the Interfacial Form of the Wiedemann-Franz Law. Phys. Rev. Lett. 2012, 108, 255901. [CrossRef]

30. Gundrum, B.C.; Cahill, D.G.; Averback, R.S. Thermal conductance of metal-metal interfaces. Phys. Rev. B 2005, 72, 245426. [CrossRef]

31. Savija, I.; Culham, J.R.; Yovanovich, M.M.; Marotta, E.E. Review of Thermal Conductance Models for Joints Incorporating Enhancement Materials. J. Thermophys. Heat Transf. 2003, 17, 43-52. [CrossRef]

32. Little, W.A. The Transport of Heat between Dissimilar Solids at Low Temperatures. Can. J. Phys. 1959, 37, 334-349. [CrossRef]

33. Swartz, E.T.; Pohl, R.O. Thermal boundary resistance. Rev. Mod. Phys. 1989, 61, 605-668. [CrossRef]

34. Reddy, P.; Castelino, K.; Majumdar, A. Diffuse mismatch model of thermal boundary conductance using exact phonon dispersion. Appl. Phys. Lett. 2005, 87, 211908. [CrossRef]

35. Fenech, H.; Rohsenow, W.M. Prediction of Thermal Conductance of Metallic Surfaces in Contact. J. Heat Transf. 1963, 85, 15-24. [CrossRef]

36. Gang, C. Ballistic-Diffusive Heat-Conduction Equations. Phys. Rev. Lett. 2001, 86, 2297-2300. [CrossRef]

37. Fletcher, L.S.; Blanchard, D.G.; Kinnear, K.P. Thermal conductance of multilayered metallic sheets. J. Thermophys. Heat Transf. 1993, 7, 120-126. [CrossRef]

38. Mayadas, A.F.; Shatzkes, M. Electrical-Resistivity Model for Polycrystalline Films: The Case of Arbitrary Reflection at External Surfaces. Phys. Rev. B 1970, 1, 1382. [CrossRef]

39. Jin, J.S.; Lee, J.S.; Kwon, O. Electron effective mean free path and thermal conductivity predictions of metallic thin films. Appl. Phys. Lett. 2008, 92, 171910. [CrossRef]

40. Nath, P.; Chopra, K.L. Thermal conductivity of copper films. Thin Solid Films 1974, 20, 53-62. [CrossRef]

41. Kelemen, F. Pulse method for the measurement of the thermal conductivity of thin films. Thin Solid Films 1976, 36, 199-203. [CrossRef]

42. Moffat, R.J. Describing the uncertainties in experimental results. Exp. Therm. Fluid Sci. 1988, 1, 3-17. [CrossRef]

Publisher's Note: MDPI stays neutral with regard to jurisdictional claims in published maps and institutional affiliations.

(C) 2020 by the authors. Licensee MDPI, Basel, Switzerland. This article is an open access article distributed under the terms and conditions of the Creative Commons Attribution (CC BY) license (http://creativecommons.org/licenses/by/4.0/). 